\title{
Evaluation of condylar cortical bone thickness in patients with different vertical facial dimensions using cone-beam computed tomography.
}

\section{Antonino Lo Giudice}

Universita degli Studi di Catania

Lorenzo Rustico ( $\square$ lorenzo.rustico@gmail.com )

University of Messina https://orcid.org/0000-0001-8529-7947

Alberto Caprioglio

Universita degli Studi dell'Insubria

Angela Mirea Bellocchio

Universita degli Studi di Messina

\section{Elda Gatto}

Universita degli Studi di Messina

\section{Marco Portelli}

Universita degli Studi di Messina

Angela Militi

Universita degli Studi di Messina

\section{Carolina Dolci}

Universita degli Studi di Messina

\section{Paola Spinuzza}

Universita degli Studi di Messina

Rosalia Leonardi

Universita degli Studi di Catania

Riccardo Nucera

Universita degli Studi di Messina

\section{Research article}

Keywords: Condylar cortical bone thickness, facial vertical skeletal pattern, muscular function, cone beam computer tomography

Posted Date: October 21st, 2019

DOI: https://doi.org/10.21203/rs.2.16254/v1 
License: (c) (i) This work is licensed under a Creative Commons Attribution 4.0 International License. Read Full License 


\section{Abstract}

Background The aim of this study is to evaluate through computed tomography differences in cortical plate thickness of condyle in patients with different facial vertical skeletal pattern.

Methods The final sample of this retrospective study included CBCT exams of 60 adult subjects (mean age $33.2 \pm 5.6$ ), selected from the digital archive of a private practice. The subjects were assigned to 3 different groups according to the values of the Frankfurt-mandibular plane angle: hyper-normo- and hypodivergent group. The volume rendering of the mandible was obtained and three condylar points were marked on it: median pole, lateral pole and the most cranial point. For each considered reference point the minimum distance between external and internal cortical surface was measured, obtaining three different outcomes: median pole, lateral pole and cranial point cortical bone thickness. The measurement was executed by means of Mimics software by the same expert operator in specific scan views.

Results The cortical bone thickness of hyperdivergent patients was found to be statistically thicker than normodivergent patients and hypodivergent patients. Cortical bone thickness of normodivergent patients was found thicker than hypodivergent patients. All the differences were statistically significant $(p<0.05)$. The Person correlation coefficient showed a statistically significant correlation $(p<.001)$ between the Frankfurt-mandibular plane angle and the evaluated cortical bone thickness outcomes.

Conclusion Facial biotype characteristics that define vertical facial skeletal pattern affect the cortical bone thickness of mandibular condyle.

\section{Background}

The interaction of masticatory muscles and craniofacial skeleton plays an important role in craniofacial growth development and masticatory system function. Several studies showed a relationship between muscle activity and vertical skeletal pattern [1-3]. Literature showed that during mandibular function and in particular during closing, biting, and chewing, the mandible can distort, bend, and stretch [4-7]. Cortical bone thickness can react directly to the load exerted by the masticatory muscles to the mandibular bone [8] or it can react indirectly to the muscular load if it is applied by the articulating surfaces of the dentition and condyles [9].

The thickness of the cortical bone responds to this functional loading, changing his characteristics. As a consequence, the evaluation of cortical bone characteristics could provide clinicians and researchers a deeper understanding of mandibular function [10-12].

Several reports showed that mandibular cortical bone thickness and mineralization can vary specifically in subjects with different vertical facial patterns [13-18].

However, to date no study evaluated mandibular cortical bone condyle thickness in patients with different vertical facial dimension patterns. 
Computed tomography (CT) is a diagnostic imaging technology that is considered the gold standard in order to investigate skeletal anatomy of oral cavity and facial complex [19]. Comparing it to conventional radiologic techniques, the use of computed tomography allows the measurement of anatomical craniofacial structures with greater accuracy [19]. In the last decades dose reduction was obtained with the use of Cone Beam Computed Tomography ( $\mathrm{CBCT}$ ) or with the introduction of Low Dose Multislice Computed Tomography protocols allowed the three-dimensional diagnostic information provided by CT become more frequently available for clinicians and researchers [20-22]. The aim of this study was to evaluate through computed tomography, the differences in cortical plate thickness of condyles in patients with different facial vertical skeletal pattern.

\section{Methods}

The sample of this retrospective study included cone beam computer tomography exams of 135 adult subjects, selected from the digital archive of a private practice. The exams were consecutively selected from a digital archive according to a specific inclusion criteria: Caucasian subjects, aged between 22 and 42y.o., have a complete permanent dentition with or without third molars, all sagittal class of skeletal and dental malocclusion were included. Exclusion criteria were: genetic syndromes or craniofacial dysmorphism, history of facial trauma and previous orthognathic surgery treatment.

The protocol of this study was approved by the Human Research Ethical Committee (103/16).

All exams were performed with i-CAT scanner (Imaging Sciences International, Hatfield, Pa) after setting the acquisition parameters as follows: $120 \mathrm{Kv}, 5 \mathrm{~mA}$ and $4-6$ second to exposure time.

The region of interest (ROI) of the exams extended from glabella to the lower mandibular border.

For each CT exam, a volume rendering of skeletal structures (Figure 1) was performed by means of a specific software (Mimics®: Materialise's Interactive Medical Images Control System, Materialise BV, Leuven, Belgium). The volume rendering was reoriented and modified applying transparency, in order to obtain a conventional latero-lateral cephalograms imagines (Figure 2). [23]

On volume-derived cephalograms conventional cephalometric analysis was performed and Frankfurtmandibular plane angle was calculated (figure 3).

The subjects were assigned to 3 different groups according to the values of the Frankfurt-mandibular plane angle. Patients with an angle greater than $27^{\circ}$ were included in the high angle facial group, others with an angle ranged from $27^{\circ}$ to $19^{\circ}$ were included in the normal angle group and patients with an angle smaller than $19^{\circ}$ were included in the low angle group.

At the end of this subdivision we obtain 26 subjects with high angle facial group, 72 patients with a mandibular plane angle ranged from $27^{\circ}$ to $19^{\circ}$, and 37 patients with a mandibular plane angle smaller than $19^{\circ}$. 
A random sequence generator (http://www.randomizer.org) was employed to generate three lists of randomized numbers of 26,72 and 37 numbers. The first 20 numbers of each random lists were selected, an adjustment was performed in order to have equal numbers of male and female in each group. Finally 60 CBCT were selected and included in the study.

Thus, the final sample of 60 subjects ( 30 female subjects and 30 male subjects) comprised 20 subjects for each facial vertical type. The cortical bone thickness of condyles of the final sample were measured according to a reproducible procedure. The volume rendering of the mandible was obtained and three condylar points were marked on it: median pole, lateral pole and the most cranial point (figure 4).

For each considered reference point the minimum distance between external and internal cortical surface was measured, obtaining three different outcomes: median pole cortical bone thickness, lateral pole cortical bone thickness and the cranial point cortical bone thickness. The measurement was executed by means of Mimics software by the same expert operator in specific scan views (Figure 5).

\section{Statistics}

A preliminary analysis on 10 subjects was conducted to evaluate the sample size. The data employed to perform the analysis were: superior pole cortical bone thickness of hyperdivergent patients $(1.37 \mathrm{~mm})$; superior pole cortical bone thickness of normodivergent patients $(1.05 \mathrm{~mm})$; pooled $\sigma(0.36)$. Power analysis results indicate that in order to reach $80 \%$ of power, it was necessary to analyze a sample of 20 CBCT scans per group. Normal distribution was evaluated through Shapiro Wilk test and the equality of variances was measured with Levene's test. Descriptive statistics has been perfoemd for each facial biotype subgroup which reported a minimum or maximum value and standard deviation of the sample. In order to evaluate the differences among the groups, Inferential statistics has been carried out. Preliminary data analysis was performed with Shapiro-Wilk test for normal distribution evaluations and with Levene's test for equality of variance evaluation. When data showed normal distribution and equality of variance, the following parametric tests were used: Analysis of variance (ANOVA), Tukey post-hoc test. In case data showed non-normal distribution, the following non-parametric tests were used: Kruskal-Wallis test and Dunn-Bonferroni post-hoc test. Inferential statistics was performed applying multiple comparisons and post hoc tests among patients with different vertical mandibular patterns (different facial bio-type) for the cortical bone thickness of evaluated condylar sites: superior pole, medial pole and lateral pole. Person correlation coefficient was calculated between the Frankfurt-mandibular plane angle and each considered cortical bone thickness.

In order to assess the methodological error, rendering landmarks selection and outcomes measurements were repeated for 10 randomly selected patients one week apart. Paired t-test and Intra-class correlation coefficient (ICC) were used to assess the intra-operator reliability. The magnitude of the random error was assessed using the Dahlberg formula. No differences $(p<0.05)$ were found between the two readings, all measurements were highly reliable with the ICC varying from 0.81 to 0.92 . Random error ranged from 0.2 
to $0.4 \mathrm{~mm}$. Statistical analysis was performed with IBM SPSS Statistics Version 20 for Windows (IBM Corporation, NY, USA. SPSS, Inc., an IBM Company), the level of significance was set at $\mathrm{P}<.05$.

\section{Results}

Table 1 reports the characteristics of the three-considered sample selected according their vertical facial pattern: hyperdivergent patients, normodivergent patients, hypodivergent patients. Male/female ratio, average age, average mandibular plane angulation and sagittal skeletal malocclusion are reported.

The Person correlation coefficient showed a statistically significant correlation $(p<.001)$ between the Frankfurt-mandibular plane angle and the evaluated cortical bone thickness outcomes (i.e. cranial Pole, medial pole, and lateral pole). The correlation coefficient ranges from -1 to 1 , we obtained for the three performed tests a strong positive correlation obtaining values comprised between 0.63 and 0.76 .

Specifically, we obtain the values of: $0.65,0.63,0.76$ respectively for the following outcomes: cranial pole, medial pole, and lateral pole.

On average the cortical bone thickness of hyperdivergent patients was found thicker than normodivergent patients and hypodivergent patients, cortical bone thickness of normodivergent patients was found thicker than hypodivergent patients. All the differences were statistically significant $(p<0.05)$.

\section{Discussion}

This is the first study that has evaluated, by means of CBCT, mandibular condylar cortical bone thickness of adult patients, comparing 3 groups with different vertical skeletal growth pattern. Other studies evaluated cortical bone of different mandibular regions of patients with different vertical patterns.

Evidence showed that patients with different vertical skeletal growth pattern produce different forces during swallowing, chewing and maximum bite force [3,24-27].

Specifically, long-face patients showed reduced occlusal forces and short-face patients showed increased occlusal force during maximum bite. This evidence confirmed that muscular function and facial morphology are related to some extend.

Different clinical studies $[16,17,28]$, performed with the aim to evaluate the relationship between different facial vertical skeletal growth types and thickness of mandibular cortical bone, demonstrate that vertical skeletal growth pattern can affect specific mandibular characteristics. These studies evaluated cortical bone thickness by mandibular cross-section scans obtained from CBCT exams in patients with different vertical facial dimensions. The results of these studies showed that long-face patients present thinner cortical mandibular bone compared to short face patient $[16,17,28,29]$.

Experimental studies seem to indicate that the cortical bone characteristics of patients with different skeletal pattern could be related to the specific muscular function [3]. Tsai and coworkers injected into the 
left masseter muscle of rats Botulinum neurotoxin type A (BTX-A) to reduce masticatory muscle function [30]. Ninety days after the rats were sacrificed, and the authors noticed next to the botulin injection a significant decrease of cortical thickness, bone mineral content and ramus height. Moreover, it was noticed on the experimental side the increase of gonial angle and crown height of the posterior teeth. Tsai and coworkers reached the conclusion that muscular function is able to affect both the morphology of the overall mandible (gonial angle dimension, ramus height) as well as bone characteristics (cortical thickness and bone mineral content).

The results of our study seemed to show that patients with increased vertical skeletal growth pattern showed a significantly greater cortical condylar bone thickness compared to patients with normal and reduced vertical skeletal growth pattern. In order to explain this finding we analyzed forces geometries produced by the masticatory muscles in patients with different vertical growth patterns (Figure 6).

In figure 6 we represented with the red color the forces produced by the masseter muscle on mandibular skeleton and teeth. We know that masticatory forces are released on the occlusion and on temporomandibular joints, so we decomposed the masticatory forces of the masseter into two forces directed towards the anatomical structure (TMJ and teeth) on which masticatory forces are directed.

Analyzing the geometry of the vectors of masticatory force and its components, it is possible to observe that the masticatory forces of high angle patients are closer to the temporomandibular joint compared to the low angle patients, consequently the component of the force acting on the condyle of high angle patients is higher in module compared to the force module acting on the condyle of low angle patients (figure 6). Moreover, for the same geometric reasons the force acting on teeth of high angle patients are decreased compared to those acting on low angle patients.

For these geometric considerations, it is possible to hypothesize, that the load on the condyle tend to be higher in patients with hi angle mandibular plane.

This hypothesis is indirectly confirmed by some evidence. Ramos and co-workers analyzed load transfer in the fossa component based on two numerical models of total temporomandibular joint implants at both 5 and $15 \mathrm{~mm}$ of mouth opening [30]. They found that the load on the TMJ at $15 \mathrm{~mm}$ of mouth opening was increased compared to the $5 \mathrm{~mm}$ mouth opening.

Moreover, Sim et al. evaluated cartilage remodeling of the mandibular condyle in young adult monkeys after an increase in vertical dimension of the midface by the use of 5,10 and $15 \mathrm{~mm}$ fixed bite-splints for 48 weeks [31]. These authors found that in the experimental animals the prechondroblastic layer (absent in control animals) was very distinctive (30-75 microns); and there was a $62 \%$ thickness augmentation in the of the chondroblastic layer. The results of this study seemed to show that the mouth opening leads to a higher ossification of condyle, this ossification could be related to the muscular function alteration consequently to the induced mandibular postural changes and to the increased TMJ load related to the biomechanical consideration previously reported. 
This is the first study in literature that evaluates the condyle cortical bone characteristics of patients with different skeletal growth patterns. The results of this study can provide new evidence about specific skeletal characteristics of patients with different muscular activity and consequently can help us understand the mechanisms that can contribute to develop skeletal malocclusions and the possible adverse effects of surgical treatments of skeletal malocclusion.

It is known that condylar resorption can occur as an unfortunate sequela of orthognathic surgery.

Kerstens et al. [32] observed radiographic evidence of condylar atrophy in 12 patients out of 206 that underwent surgical orthodontic treatment. All 12 patients had the same dentofacial deformity: high-angle mandibular retrognathia. They considered the dentofacial deformity to be the main reason for condylar resorption and hypothesized that orthognathic surgery acts as a factor that stimulates the progress of the disease by increasing the load on TMJs.

The results of this study and the explanation provided by its authors can help explain the cortical differences observed among patients with different vertical skeletal pattern, and they could help orthognathic surgeons and orthodontists to evaluate the role of masticatory function in patients with different skeletal characteristics.

Different aspects must be taken into consideration when evaluating the results of this investigation including its limitations. This study did not evaluate the possible differences between male and female subjects. However, in a recent study by Farnsworth et al., no sex differences in cortical bone thickness in the mandible were found between both sexes [33].

Limitations of CBCT imaging should also be considered in terms of spatial resolution and in terms of bone density evaluation [34]. CBCT images do not have consistent Hounsfield values and consequently it does not provide an accurate bone density evaluation [35]. Further research will be necessary in order to directly correlate cortical condylar bone and masticatory function.

\section{Conclusions}

-Facial biotype characteristics that define vertical facial skeletal pattern affect the cortical bone thickness of mandibular condyle.

-Patients with increased vertical skeletal growth pattern showed a significantly greater cortical condylar bone thickness compared to patients with normal and reduced vertical skeletal growth pattern. Patients with normal vertical skeletal growth pattern showed a significantly greater cortical condylar bone thickness compared to patients with reduced vertical skeletal growth pattern.

\section{Abbreviations}

- CT: Computed tomography 
- CBCT: Cone Beam Computed Tomography

- ROI: Region of interest

- FMA: Frankfurt-mandibular-angle

- ANOVA: Analysis of variance

- ICC: Intra-class correlation coefficient

- TMJ: Temporomandibular joint

\section{Declarations}

- Ethics approval and consent to participate: The protocol of this study was approved by the Human Research Ethical Committee of the University Hospital "G. Martino" (Via Consolare Valeria, 1-98125 Messina. aoucomitatoetico@unime.it) approved with reference number 103/16 during the committee meeting of the 21st of November 2016.

- Consent for publication: Not applicable

- Availability of data and materials: The datasets used and/or analysed during the current study are available from the corresponding author on reasonable request.

- Competing interests: The datasets used and/or analysed during the current study are available from the corresponding author on reasonable request. No competing interests to declare.

- Funding: No source of funding.

- Authors' contributions: RN \& ALG had designed the study; AMB, EG, PS \& CD had acquisited the CBCT data, analyzed and selected them; ALG, PS \& RL performed the volume rendering of the CBCT; ALG \& LR performed the measurements on the CBCT rendering; AM \& MP performed the statistical analysis; ALG \& LR had dradted the work; RN \& AC had revised the work. All authors read and approved the final manuscript.

- Acknowledgements: Not applicable.

\section{References}

1. Akada, K.; Lowe, A.A.; Freund, V.K. Canonical correlations between masticatory muscle orientation and dentoskeletal morphology in children. Am J Orthod Orofacial Orthop 1984, 86, 331-41, DOI: https://doi.org/10.1016/0002-9416(84)90144-1.

2. van Spronsen, P.H.; Weijs, W.A.; Valk, J.; Prahl-Andersen, B.; van Ginkel, F.C. A comparison of jaw muscle cross-sections of long-face and normal adults. J Dent Res 1992, 7, 1279-85, DOI: 10.1177/00220345920710060301.

3. Proffit, W.R., Fields, H.W.; Nixon, W.L. Occlusal forces in normal- and long-face adults. J Dent Res. 1983, 62, 566-70, DOI: 10.1177/00220345830620051301. 
4. Korioth, T.W.; Hannam, A.G. Deformation of the human mandible during simulated tooth clenching. J Dent Res 1994, 73, 56-66, DOI: 10.1177/00220345940730010801.

5. Korioth, T.W.; Romilly, D.P.; Hannam, A.G. Three-dimensional finite element stress analysis of the dentate human mandible. Am J Phys Anthropol 1992, 88, 69-96 DOI: 10.1002/ajpa.1330880107.

6. Cattaneo, P.M.; Dalstra, M.; Melsen, B. The transfer of occlusal forces through the maxillary molars: a finite element study. Am J Orthod Dentofacial Orthop 2003, 123, 367-73, DOI: 10.1067/mod.2003.73

7. Usui, T.; Maki, K.; Toki, Y.; Shibasaki, Y.; Takanobu, H.; Takanishi, A.; Hatcher, D.; Miller, A. Measurement of mechanical strain on mandibular surface with mastication robot: influence of muscle loading direction and magnitude. Orthod Craniofac Res 2003; 6 (Suppl 1), 163-7, DOI: 10.1034/j.1600-0544.2003.250.x.

8. Hylander, W.L.; Johnson, K.R.; Crompton, A.W. Muscle force recruitment and biomechanical modeling: an analysis of masseter muscle function during mastication in Macaca fascicularis. Am J Phys Anthropol 1992, 88, 365-87, DOI: 10.1002/ajpa.1330880309.

9. Hylander, W.L.; Johnson, K.R.; Crompton, A.W. Loading patterns and jaw movements during mastication in Macaca fascicularis: a bone-strain, electromyographic, and cineradiographic analysis. Am J Phys Anthropol 1987, 72, 287-314, DOI: 10.1002/ajpa.1330720304.

10. Hylander, W.L. In-vivo bone strain as an indicator of masticatory bite force in Macaca fascicularis. Arch Oral Biol 1986, 31, 149-57, DOI: 10.1016/0003-9969(86)90120-2.

11. Hylander, W.L. Stress and strain in the mandibular symphysis of primates: a test of competing hypotheses. Am J Phys Anthropol 1984, 64, 1-46, DOI: 10.1002/ajpa.1330640102.

12. Schwartz-Dabney, C.L., Dechow, P.C. Variations in cortical material properties throughout the human dentate mandible. Am J Phys Anthropol 2003, 120, 252-77, DOI: 10.1002/ajpa.10121.

13. Maki, K.; Miller, A.; Okano, T.; Shibasaki, Y. Changes in cortical bone mineralization in the developing mandible: a three-dimensional quantitative computed tomography study. J Bone Miner Res 2000, 15, 700-9. DOI: $10.1359 / j b m r .2000 .15 .4 .700$.

14. Maki, K.; Miller, A.J.; Okano, T.; Shibasaki, Y. A three-dimensional, quantitative computed tomographic study of changes in distribu- tion of bone mineralization in the developing human mandible. Arch Oral Biol 2001, 46, 667-78, DOI: 10.1016/S0003-9969(01)00006-1.

15. Swasty, D.; Lee, J.; Huang, C.J.; Maki, K.; Gansky, S.A.; Hatcher, D.; Miller, A.J. Cross-sectional human mandibular morphology as assessed in vivo by cone-beam computed tomography in patients with different vertical facial dimensions. Am J Orthod Orofacial Orthop 2011, 139, e377-e389, DOI: 10.1016/j.ajodo.2009.10.039.

16. Masumoto, T.; Hayashi, I.; Kawamura, A.; Tanaka, K.; Kasai, K. Relationships among facial type, buccolingual molar inclination, and cortical bone thickness of the mandible. EurJ Orthod 2001, 23, 15-23, DOI: $10.1093 /$ ejo/23.1.15. 
17. Tsunori, M.; Mashita, M.; Kasai, K. Relationship between facial types and tooth and bone characteristics of the mandible obtained by CT scanning. Angle Orthod, 1998, 68, 557-62. DOI: 10.1043/00033219(1998)068<0557:RBFTAT>2.3.CO;2.

18. Fastuca, R.; Meneghel, M.; Zecca, P.A.; Mangano, F.; Antonello, M.; Nucera, R.; Caprioglio, A. Multimodal airway evaluation in growing patients after rapid maxillary expansion. Eur J Paediatr Dent 2015, 16,129-34. DOI: 10.1016/j.ijporl.2013.10.011.

19. Howerton, W.B. Jr; Mora, M.A. Advancements in digital imaging: what is new and on the horizon? Am Dent Assoc. 2008, 139 Suppl, 20S-24S, DOI: 10.14219/jada.archive.2008.0354.

20. Ludlow, J.B.; Davies-Ludlow, L.E.; Brooks, S.L. Dosimetry of two extraoral direct digital imaging devices: NewTom cone beam CT and Orthophos Plus DS panoramic unit. Dentomaxillofac Radiol 2003, 32, 229-34, DOI: $10.1259 / \mathrm{dmfr} / 26310390$.

21. Cordasco, G.; Portelli, M.; Militi, A.; Nucera, R.; Lo Giudice, A.; Gatto, E.; Lucchese, A. Low-dose protocol of the spiral CT in orthodontics: comparative evaluation of entrance skin dose with traditional X-ray techniques. Prog Orthod 2013, 10, 14:24, DOI: 10.1186/2196-1042-14-24.

22. Martina, R.; Cioffi, I.; Farella, M.; Leone, P.; Manzo, P.; Matarese, G.; Portelli, M.; Nucera, R.; Cordasco, G. Transverse changes determined by rapid and slow maxillary expansion--a low-dose CT-based randomized controlled trial. Orthod Craniofac Res 2012, 15, 159-68. DOI: 10.1111/j.1601-6343.2012.01543.x.

23. Nucera R, Lo Giudice A, Bellocchio AM, Spinuzza P, Caprioglio A, Perillo L, Matarese G, Cordasco G Bone and cortical bone thickness of mandibular buccal shelf for mini-screw insertion in adults. Angle Orthod. 2017 Sep;87(5):745-751.

24. Szymańska, J.; Sidorowicz, Ł. The relationship between selected parameters of a cephalometric analysis determining the vertical morphology of facial skeleton and bite force. Folia Morphol (Warsz) 2017 May 29, DOI: $10.5603 / F M . a 2017.0040$.

25. Gatto, E.; Matarese, G.; Di Bella, G.; Nucera R.; Borsellino C.; Cordasco G. Load-deflection characteristics of superelastic and thermal nickel-titanium wires. Eur J Orthod. 2013, 35, 115-23. DOI: 10.1093/ejo/cjr103.

26. Serrao, G.; Sforza, C.; Dellavia, C.; Antinori, M.; Ferrario, V.F. Relation between vertical facial morphology and jaw muscle activity in healthy young men. Prog Orthod 2003, 4, 45-51, DOI: 10.1034/j.16009975.2002.02031.x.

27. Lo Giudice A, Nucera R, Matarese G, Portelli M, Cervino G, Lo Giudice G, Militi A, Caccianiga G, Cicciù M, Cordasco G. Analysis of resistance to sliding expressed during first order correction with conventional and self-ligating brackets: an in-vitro study. Int J Clin Exp Med 2016; 9 (8):

28. Sadek, M.M.; Sabet, N.E.; Hassan, I.T. Three-dimensional mapping of cortical bone thickness in subjects with different vertical facial dimensions. Prog Orthod 2016, 17, 32, DOI: 10.1186/s40510-016-0145-x.

29. Nucera, R.; Lo Giudice, A.; Matarese G.; Artemisia A.; Bramanti E.; Crupi P.; Cordasco G. Analysis of the characteristics of slot design affecting resistance to sliding during active archwire configurations. Prog 
Orthod 2013, 14, 35. DOI: 10.1186/2196-1042-14-35.

30. Tsai, C.Y.; Huang, R.Y.; Lee, C.M.; Hsiao, W.T.; Yang, L.Y. Morphologic and bony structural changes in the mandible after a unilateral injection of botulinum neurotoxin in adult rats. J Oral Maxillofac Surg 2010, 68, 1081-7. DOI: 10.1016/j.joms.2009.12.009.

31. Ramos, A.; Mesnard, M. Load transfer in Christensen( $\left.{ }^{\circledR}\right)$ TMJ in alloplastic total joint replacement for two different mouth apertures. J Craniomaxillofac Surg 2014, 42, 1442-9, DOI: 10.1016/j.jcms.2014.04.008.

32. Sim, Y.; Carlson, D.S.; McNamara, J.A. Condylar adaptation after alteration of vertical dimension in adult rhesus monkeys, Macaca mulatta. Cranio. 1995, 13, 182-7, DOI: 10.1080/08869634.1995.11678066.

33. Kerstens, H.C.; Tuinzing, D.B.; Golding, R.P.; van der Kwast, W.A. Condylar atrophy and osteoarthrosis after bimaxillary surgery. Oral Surg Oral Med Oral Pathol. 1990, 69, 274-80, DOI: 10.1016/0030-4220(90)902862.

34. Farnsworth, D.; Rossouw, P.E.; Ceen, R.F.; Buschang, P.H. Cortical bone thickness at common miniscrew implant placement sites. Am J Orthod Dentofacial Orthop 2011,139, 495-503. DOI: 10.1016/j.ajodo.2009.03.057.

35. Molen, A.D. Considerations in the use of cone-beam computed tomography for buccal bone measurements. Am J Orthod Den tofacial Orthop 2010, 137, S130-5, DOI: 10.1016/j.ajodo.2010.01.015.

\section{Tables}




\begin{tabular}{|c|c|c|c|c|c|c|}
\hline & $\begin{array}{c}\text { Male/female } \\
\text { Subjects }\end{array}$ & $\begin{array}{l}\text { Mean } \\
\text { Age } \\
\pm \mathrm{SD}\end{array}$ & 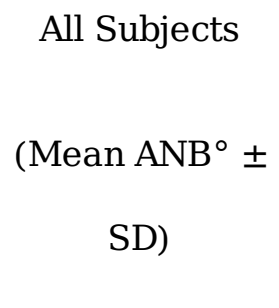 & $\begin{array}{c}\text { Class I } \\
\text { subjects } \\
\text { (Mean ANB } \pm \\
\text { SD) }\end{array}$ & $\begin{array}{c}\text { Class II } \\
\text { subjects } \\
\text { (Mean } \mathrm{ANB}^{\circ} \pm \\
\mathrm{SD})\end{array}$ & $\begin{array}{c}\text { Class III } \\
\text { subjects } \\
\text { (Mean } \mathrm{ANB}^{\circ} \pm \\
\mathrm{SD})\end{array}$ \\
\hline $\begin{array}{l}\text { ivergent } \\
\text { ents }\end{array}$ & $10 / 10$ & $\begin{array}{c}33.3 \pm \\
5.3\end{array}$ & $20(2.5 \pm 2.5)$ & $13(1.9 \pm 1.1)$ & $5(5.9 \pm 0.8)$ & $2(-1.8 \pm 0.5)$ \\
\hline $\begin{array}{l}\text { ivergent } \\
\text { ents }\end{array}$ & $8 / 12$ & $\begin{array}{c}32.5 \pm \\
5.5\end{array}$ & $20(2.6 \pm 2.7)$ & $11(2.1 \pm 1.2)$ & $6(5.5 \pm 1.0)$ & $3(-2.1 \pm 1.4)$ \\
\hline \multirow[t]{3}{*}{ vergent } & $12 / 8$ & $\begin{array}{c}33.9 \pm \\
6.3\end{array}$ & $20(2.3 \pm 2.9)$ & $12(2.3 \pm 1.0)$ & $5(5.7 \pm 1.0)$ & $3(-2.7 \pm 0.5)$ \\
\hline & $30 / 30$ & $\begin{array}{c}33.2 \pm \\
5.6\end{array}$ & $60(2.5 \pm 2.7)$ & $36(2.1 \pm 1.1)$ & $16(5.7 \pm 0.9)$ & $8(-2.3 \pm 0.7)$ \\
\hline & & $\mathrm{NS}^{\dagger+}$ & $\mathrm{NS}^{+\dagger}$ & $\mathrm{NS}^{\dagger \dagger}$ & $\mathrm{NS}^{\dagger \dagger}$ & $\mathrm{NS}^{\dagger+}$ \\
\hline
\end{tabular}

Table 1. Samples characteristics. ${ }^{\dagger}$ Analysis of Variance. ${ }^{\dagger \dagger}$ Not significant.

Table 2 shows descriptive statics of considered condylar cortical bone outcomes in the three considered samples.

\begin{tabular}{|c|c|c|c|c|c|c|c|c|c|c|c|c|}
\hline & \multicolumn{4}{|c|}{ Superior pole } & \multicolumn{5}{|c|}{ Medial pole } & \multicolumn{3}{|c|}{ Lateral Pole } \\
\hline & Mean & SD & Min & Max & Mean & SD & Min & Max & Mean & SD & Min & Max \\
\hline $\begin{array}{l}\text { Hyperdivergent } \\
\text { patients }\end{array}$ & 1.34 & 0.27 & 0.70 & 1.80 & 1.42 & 0.41 & 0.93 & 2.34 & 1.36 & 0.29 & 0.98 & 1.89 \\
\hline $\begin{array}{l}\text { Normodivergent } \\
\text { patients }\end{array}$ & 1.05 & 0.12 & 0.87 & 1.40 & 0.92 & 0.16 & 0.65 & 1.26 & 1.07 & 0.14 & 0.80 & 1.43 \\
\hline $\begin{array}{l}\text { Hypodivergent } \\
\text { patients }\end{array}$ & 0.85 & 0.20 & 0.47 & 1.38 & 0.81 & 0.12 & 0.60 & 1.08 & 0.81 & 0.10 & 0.63 & 0.95 \\
\hline
\end{tabular}


Table 2. Descriptive statistics of cortical condyle bone thickness. Mean value, standard deviation, minimum and maximum values were reported for each evaluated anatomical condylar site (i.e. the superior pole, the medial pole and the lateral pole).

Table 3 reports inferential statistics performed comparing the different groups and the relative applied tests.

\begin{tabular}{|c|c|c|c|c|}
\hline \multicolumn{2}{|c|}{ Cortical bone tickness } & Superior Pole ${ }^{\dagger}$ & Medial Pole ${ }^{\dagger \dagger}$ & Lateral Pole \\
\hline \multicolumn{2}{|c|}{ Shapiro-Wilk Normality Test } & $\mathrm{p}=.059(\mathrm{NS})$ & $\mathrm{p}<.001$ & $\mathrm{p}<.001$ \\
\hline \multicolumn{2}{|l|}{ Levene Test } & $\mathrm{p}=.034(\mathrm{NS})$ & $\mathrm{p}<.001$ & $\mathrm{p}<.001$ \\
\hline \multicolumn{2}{|c|}{ Multiple comparison tests significance } & $\mathrm{p}<.001^{* *}$ & $\mathrm{p}<.001^{*}$ & $\mathrm{p}<.001 *$ \\
\hline \multicolumn{5}{|c|}{ Post hoc comparisons: } \\
\hline Hyperdivergent & Vs. Normodivergent & $\mathrm{p}<.001 * * * *$ & $\mathrm{p}<.001 * * *$ & $\mathrm{p}<.001 * * *$ \\
\hline Hyperdivergent & Vs. Hypodivergent & $\mathrm{p}<.05^{* * * *}$ & $\mathrm{p}<.001 * * *$ & $\mathrm{p}<.001 * * *$ \\
\hline Normodivergent & Vs. Hypodivergent & $\mathrm{p}<.001 * * * *$ & $\mathrm{p}=.373 * * *(\mathrm{NS})$ & $\mathrm{p}<.001 * * *$ \\
\hline
\end{tabular}

Table 3. Multiple comparisons and post hoc tests among different evaluated sites evaluating cortical bone thickness: ${ }^{\dagger}$ Upper pole, ${ }^{\dagger \dagger}$ Medial pole, ${ }^{\dagger \dagger}$ Lateral pole. ${ }^{*}$ Analysis of variance (ANOVA); $* *$ Kruskal-Wallis test; $* * *$ Tukey test; **** Dunn-Bonferroni test.

\section{Figures}




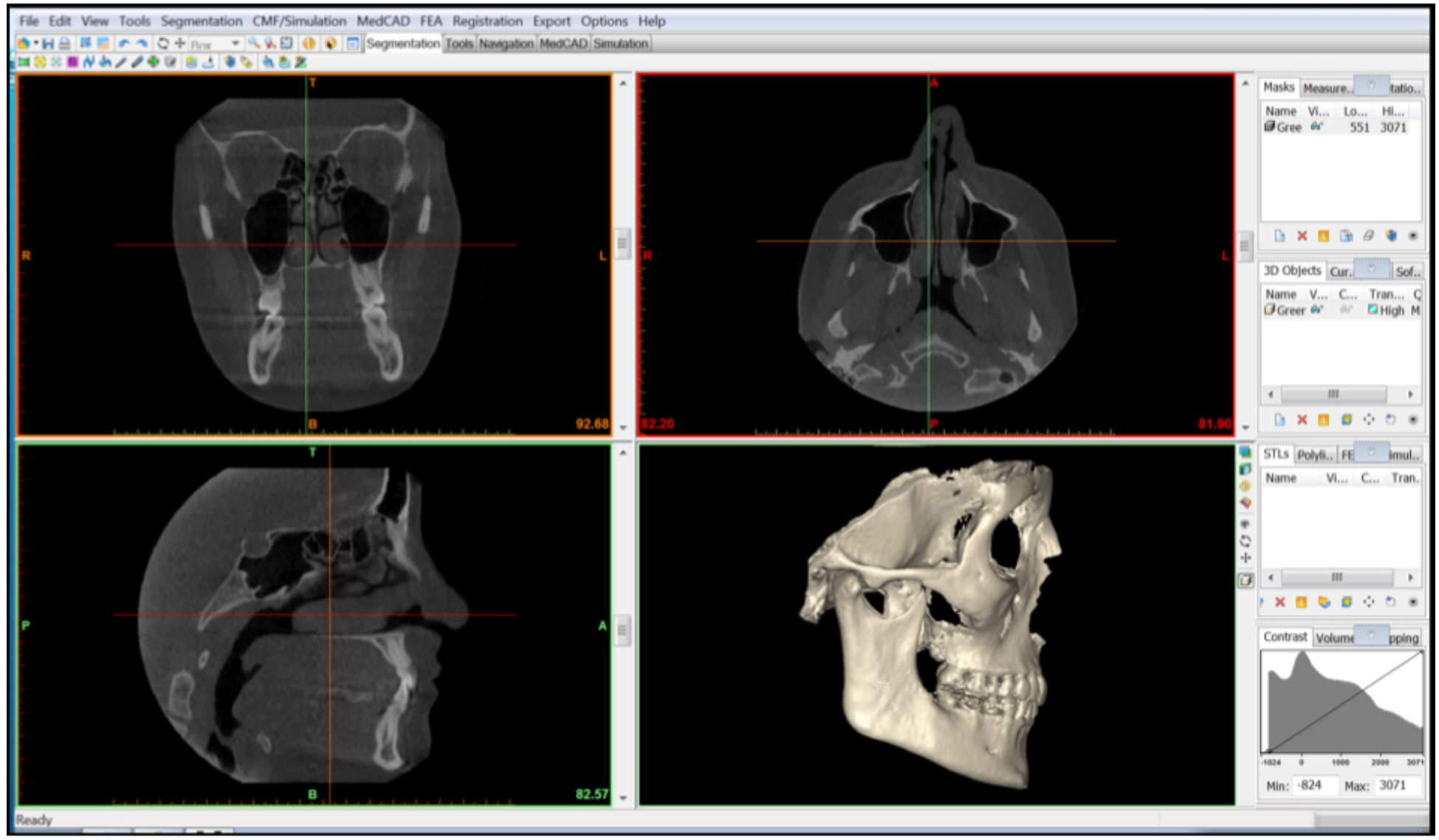

Figure 1

Volume rendering of Region of Interest. 


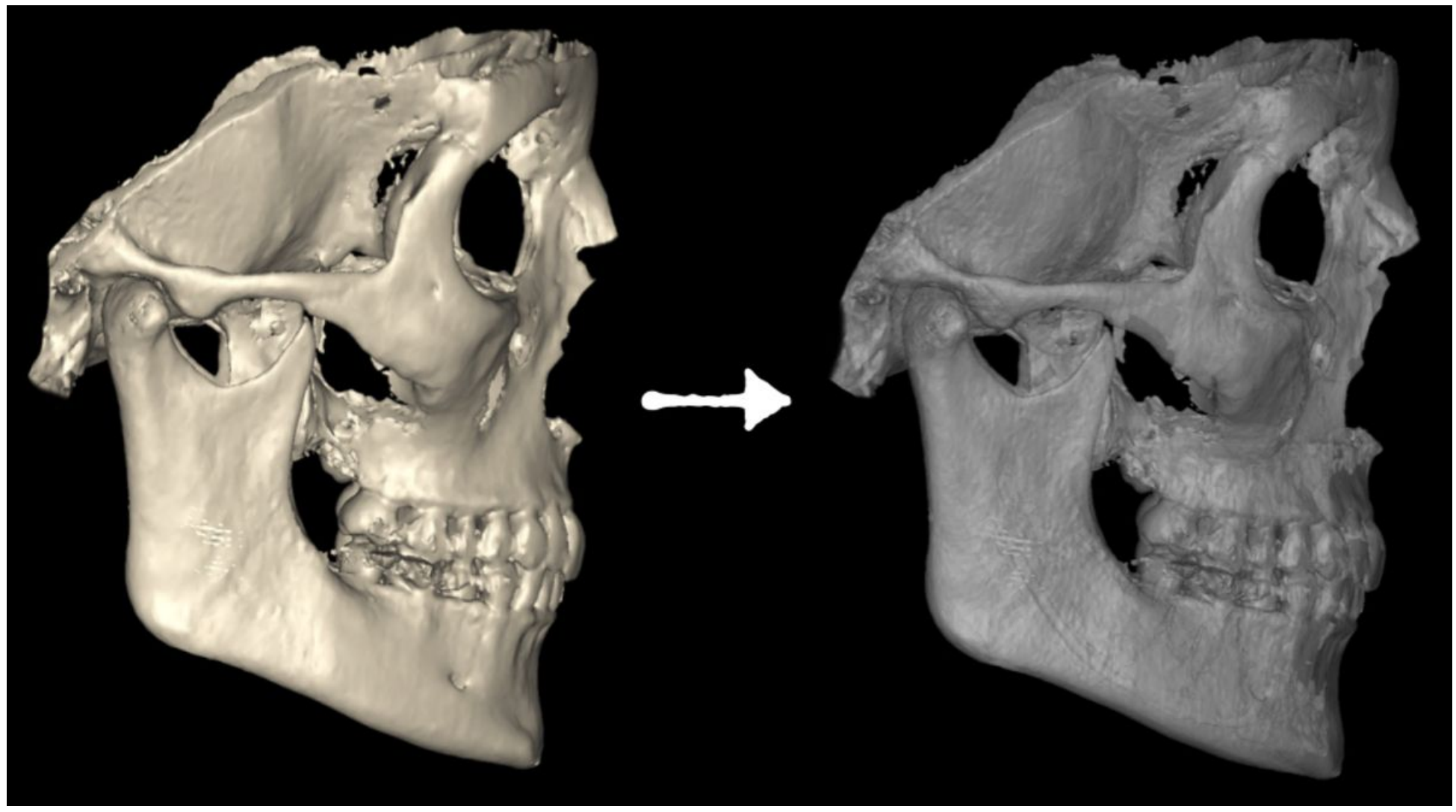

\section{Figure 2}

Transparency was applied to create a conventional radiographic cephalogram in latero-lateral projection imagines.

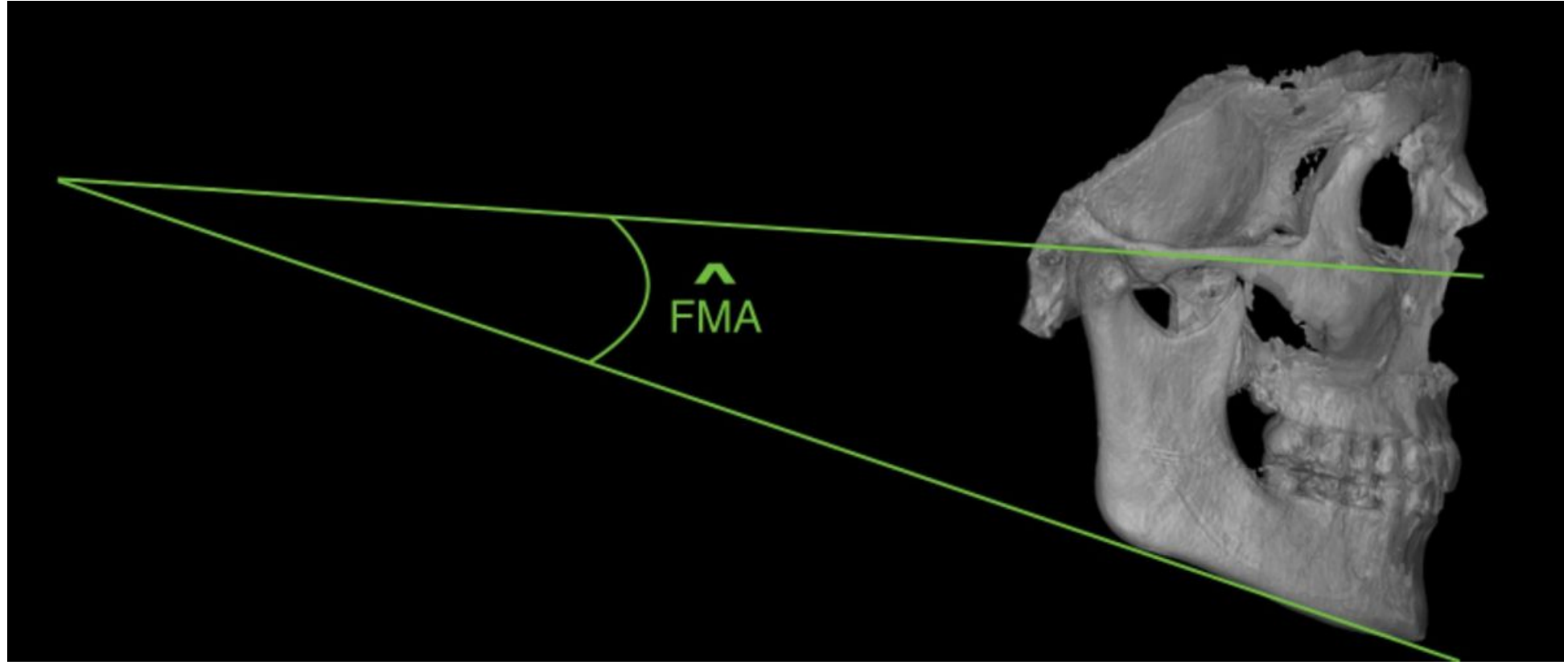

Figure 3

Frankfurt-mandibular plane calculation. 


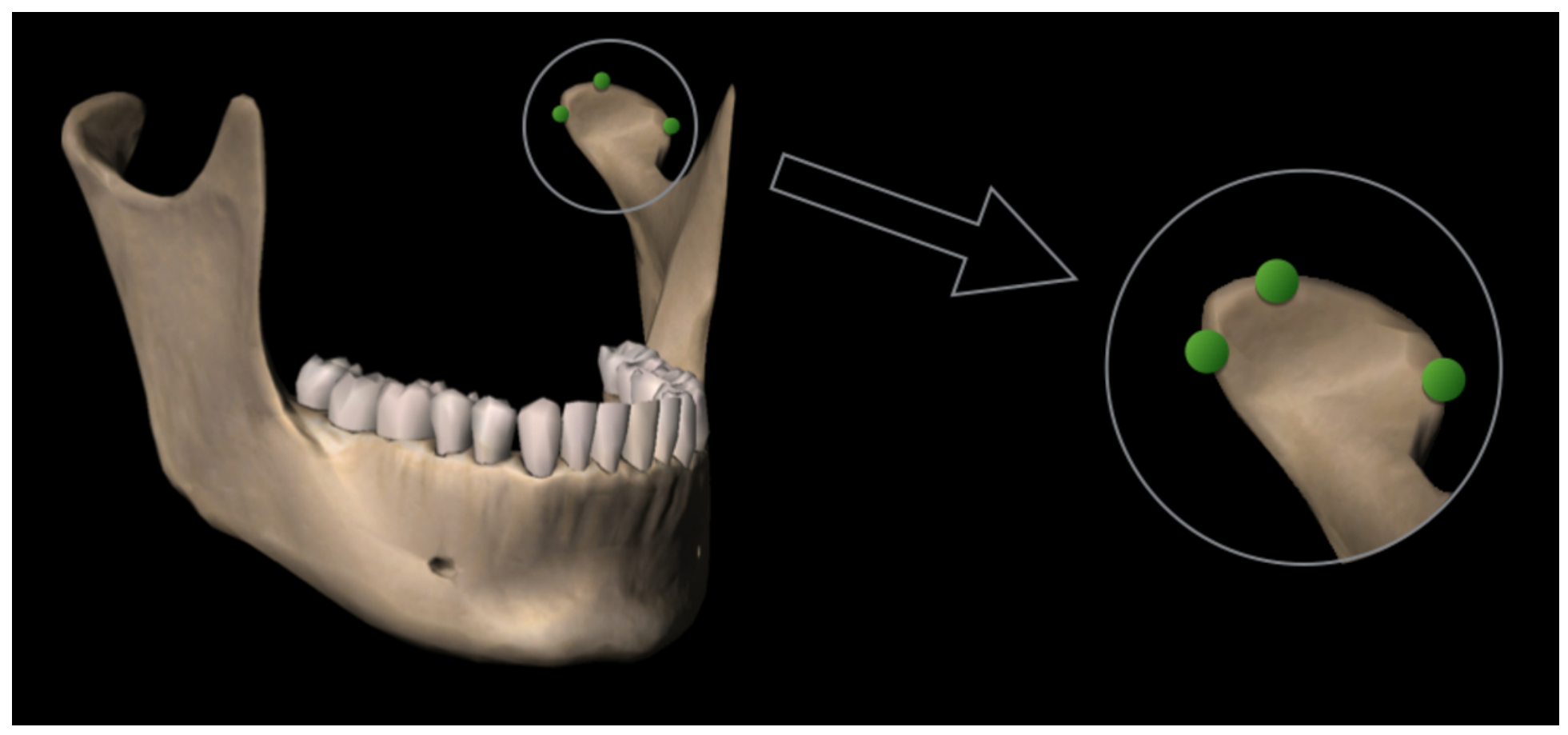

\section{Figure 4}

Condylar rendering and landmarks selection.
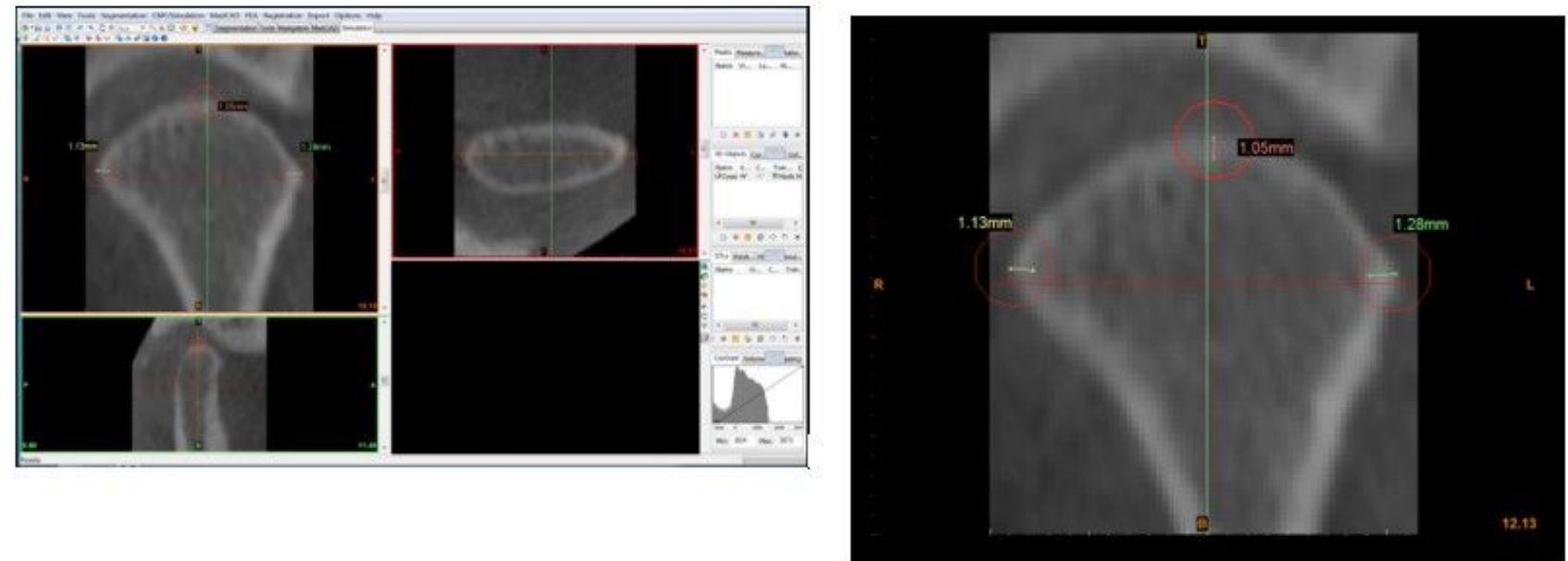

(a)

(b)

\section{Figure 5}

Landmarks were checked in proper view scans passing through the main axes of each condyle (a) and measurement were performed on the cutting scan plane passing through the long axes of mandibular condyle (b). 

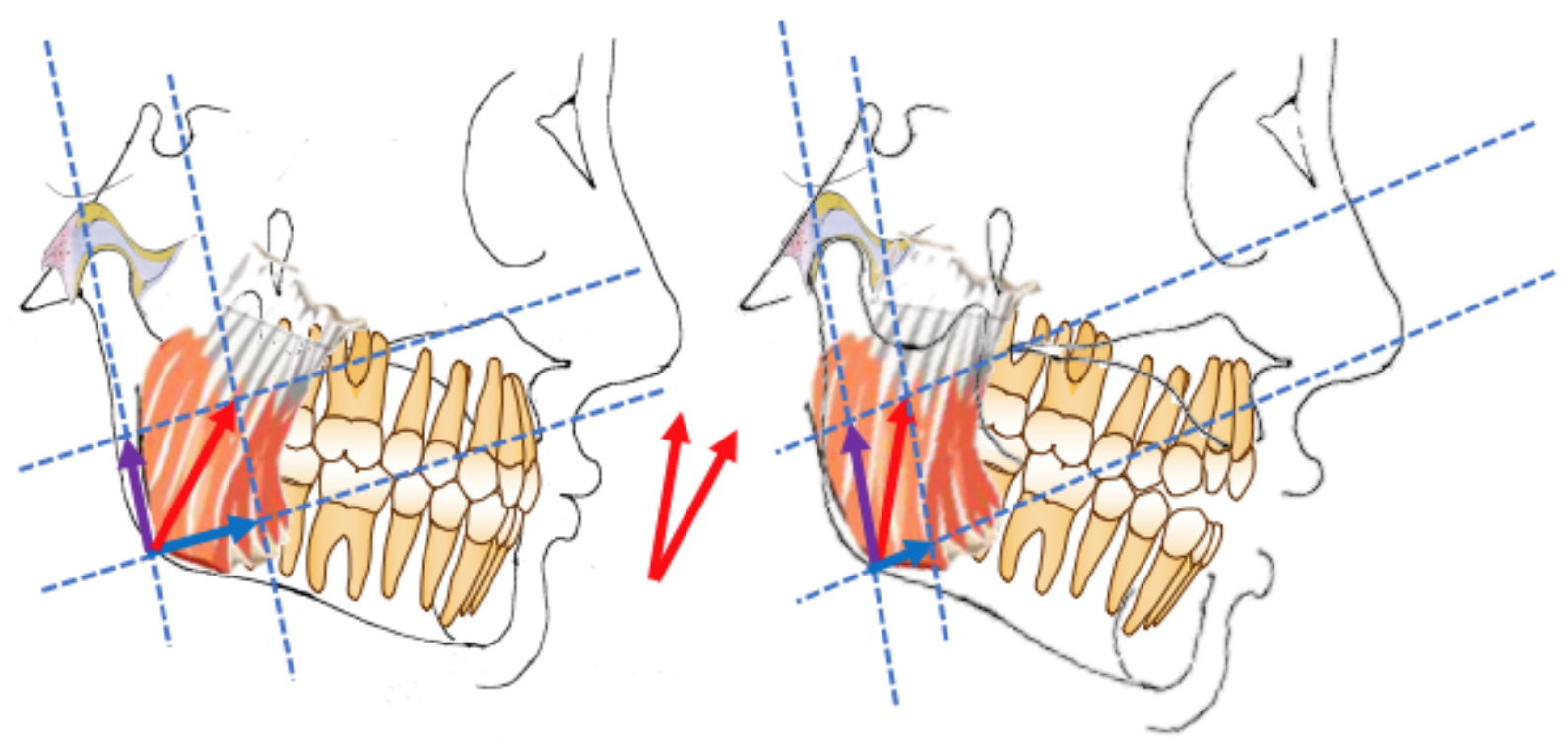

Figure 6

Forces geometries produced by masticatory muscle in patients with different vertical growth pattern. Low angle mandibular patients (A), Hi angle mandibular patient (B). The force of masseter was decomposed into two forces directed towards TMJ and teeth (for teeth a direction a line passing through the center of mandibular first molars was represented). 